\title{
Model Predictive Control of Combined Irrigation and Water Supply Systems: Application to the Guadiana River
}

\author{
V. Puig*, C. Ocampo-Martinez \\ Advanced Control Systems Group \\ IRI (CSIC-UPC) \\ Technical University of Catalonia \\ Llorens i Artigas, 4-6 \\ 08028 Barcelona, Spain
}

\author{
(Invited Paper) \\ J. Romera, J.Quevedo \\ Advanced Control Systems Group \\ Technical University of Catalonia \\ Rambla Sant Nebridi, 10 \\ 08222 Terrassa, Spain \\ Rudy Negenborn \\ Dept. of Marine and Transport Technology \\ Delft University of Technology \\ Mekelweg 2, 2628 CD Delft \\ The Netherlands
}

\author{
P. Rodríguez, S. de Campos \\ ADASA Sistemas \\ José Agustín Goytisolo, 30-32 \\ 08908 Hospitalet de Llobregat \\ Spain
}

\begin{abstract}
In this paper, a methodology for the optimal management of a combined irrigation and water supply system based on model predictive control (MPC) is proposed. A controloriented modelling methodology for this type of systems is presented as well. MPC is used to generate flow control strategies from the sources to the consumer and irrigation areas to meet future demands with appropriate flows, optimizing operational goals such as network safety volumes in dams and flow control stability in actuators (valves, gates and pumps). The case study of Guadiana river is used to exemplify and verify the proposed optimal management methodology. Results have shown the effectiveness of the proposed modelling and control methodologies.
\end{abstract}

\section{INTRODUCTION}

Water management is a subject of increasing concern. Limited water supplies, conservation and sustainability policies, as well as the infrastructure complexity for meeting consumer and irrigation demands with quality levels make water management a challenging control problem. Water supply, treatment, transport and distribution are often operated separately, by different authorities. Planning and management of these subsystems have different goals and time-scales. Additionally, the hydraulics involved differ considerably from one another, in particular, between large, spatially-distributed open channel areas and pressurized water sections for distribution to consumers. In many water systems, network operation is carried out based on heuristic approaches, operator judgment, etc., which may be very complex in large-scale interconnected systems. Decision support systems, which are based on mathematical network and operation models, may efficiently contribute

This research has been partially founded by CDTI (MCyT) project HIDROPTIM IDI-20100722.

*Corresponding author: V. Puig \{vicenc.puig@upc.edu\} to the optimal management of water networks by computing control strategies ahead in time, which optimize management goals [2]. Optimization and optimal control techniques provide an important contribution to strategy computation in systems water management, as reported in [1].

Model predictive control (MPC) provides suitable techniques to compute optimal control strategies ahead in time for all the control elements of a water system [5]. The optimal strategies are computed by optimizing a mathematical function describing the operational goals in a given time horizon and using a representative model of the network dynamics, as well as demand forecasts.

Water systems are usually comprised of:

- Supplies, where raw water is drawn from superficial or underground sources, such as rivers, reservoirs or boreholes

- Production facilities, where water is treated to meet consumer-use standards

- Transport systems, channels and other natural or artificial open-flow conduits carrying water from the sources to the treatment sites and to the distribution areas

- Distribution areas, including consumer demands, storage tanks and pressurized pipe networks, to which water must be supplied with appropriate pressure levels

- Control elements in all the above-mentioned subsystems, such as gates, valves, and pumps. Many modern water systems are operated through centralized or distributed telemetry and telecontrol.

In this paper, a methodology for the optimal management of a combined irrigation and water supply system based on MPC is proposed. A control-oriented modelling methodology 
for these systems is presented too. MPC is used to generate flow control strategies from the sources to the consumer and irrigation areas to meet future demands with appropriate flows, optimizing operational goals such as network safety volumes in dams and flow control stability in actuators. The case study of Guadiana river is presented to exemplify and verify the proposed management methodology.

The paper is organized as follows. In Section 2, the controloriented modelling methodology is described. In Section 3, the model predictive/optimal control of combined irrigation and water supply systems is presented. In Section 4, the considered case study (Guadiana river) is presented as well as the results of the application of the proposed modelling and control approach. Finally, in Section 5, the main conclusions are presented.

\section{Control Oriented Modelling Methodology}

Several modelling techniques dealing with the operational control of water systems have been presented in the literature, see [1], [6], among others. Here, a control-oriented modelling approach is outlined, which follows the principles presented by the authors in [3], [7]. The extension to include the pressuremodel can be found in [1], [6]. A water system generally contains tanks, which store the drinking water that comes from the network sources, a network of pressurized pipes and a number of sinks. Valves and/or pumping stations are elements that allow to manipulate the water flow according to a specific policy and to supply water requested by the network users. These flows are chosen by a global management strategy.

The water system model can be considered as composed of a set of constitutive elements, which are presented and discussed below.

1) Tanks and dams: Water tanks/dams provide the entire water network with the storage capacity of drinking water at appropiate elevation levels to provide adequate pressure service to consumers. The mass balance expression relating the stored volume $v$, the manipulated inflows $q_{\text {in }}^{j}$ and outflows $q_{\text {out }}^{h}$ (including the demand flows as outflows) for the $i$-th tank can be written as the discrete-time difference equation

$$
v_{i}(k+1)=v_{i}(k)+\Delta t\left(\sum_{j} q_{\text {in }}^{j}(k)-\sum_{h} q_{\text {out }}^{h}(k)\right),
$$

where $\Delta t$ denotes the sampling time and $k$ denotes the discrete-time instant. The physical constraint related to the range of admissible water in the $i$-th tank is expressed as

$$
\underline{v}_{i} \leq v_{i}(k) \leq \bar{v}_{i}, \quad \text { for all } k,
$$

where $\underline{v}_{i}$ and $\bar{v}_{i}$ denote the minimum and the maximum admissible storage capacity, respectively. As this constraint is physical, it is impossible to send more water to a tank than it can store, or drawing more water than the stored amount. Although $\underline{v}_{i}$ might correspond with an empty tank, in practice this value can be set as nonzero in order to maintain an emergency stored volume enough to supply for facing extreme circumstances. Moreover, there exists restrictions in the amount of flow that can be extracted from the tank/dam depending on the volume stored according to the discharge curves.

For simplicity purposes, the dynamic behavior of these elements is described as a function the volume. However, in most of the cases the measured variable is the tank water level (by using level sensors), which implies the computation of the water volume taking into account the tank geometry.

2) Actuators: Two types of control actuators are considered: valves/gates and pumps (more precisely, complex pumping stations). The manipulated flows through the actuators represent the manipulated variables, denoted as $q_{u}$. Both pumps and valves/gates have lower and upper physical limits, which are taken into account as system constraints. As in (2), they are expressed as

$$
\underline{q u}_{i} \leq q_{u_{i}}(k) \leq \overline{q u}_{i}, \quad \text { for all } k
$$

where $\underline{q u}_{i}$ and $\bar{q}_{u}$ denote the minimum and the maximum flow capacity, respectively. It is assumed that there is a local controller, which ensures that the required flow through the actuator is satisfied.

3) Nodes: These elements correspond to the network points where water flows are merged or split. Thus, the nodes represent mass balance relations, being modelled as equality constraints related to inflows (from other tanks/dams through valves or pumps) and outflows, these latter being represented not only by manipulated flows but also by demand flows. The expression of the mass conservation in these elements can be written as

$$
\sum_{j} q_{\text {in }}^{j}(k)=\sum_{h} q_{\text {out }}^{h}(k) .
$$

From now on and with some abuse of notation, node inflows and outflows are still denoted by $q_{\text {in }}$ and $q_{\text {out }}$, respectively, despite they can be manipulated flows and hence denoted by $q_{u}$, if correspond.

4) River reaches: A single canal reach can be approximated by using the IDZ model (see [4]) given by

$$
Y_{d n s}(s)=G_{1}(s) Q_{u p s}(s)+G_{2}(s) Q_{d n s}(s),
$$

where $Y_{d n s}(s)$ is the water level at the control point, and $Q_{u p s}(s), Q_{d s n}(s)$ are the upstream and downstream flows, respectively. Moreover, $G_{1}(s)=e^{-\tau_{d} s} / A_{d} s$ and $G_{2}(s)=$ $-1 / A_{d} s$ with $\tau_{d}$ being the downstream transport delay and $A_{d}$ the downstream backwater area.

Taking into account the linearised relation between $Q_{d n s}$ and $Y_{d n s}$ in the control point, the following relation can be established:

$$
q_{d n s}(s)=\beta Y_{d n s}(s),
$$

where $\beta$ is a constant varying with the operating point. Combining (5) and (6), the following first order plus time delay (FOPTD) model is obtained

$$
G(s)=\frac{Q_{d n s}(s)}{Q_{u p s}(s)}=\frac{K e^{-\tau_{d} s}}{T s+1},
$$


with $K=1$ and $T=A_{d} / \beta$. This model can be represented in discrete-time, using a sampling time $\Delta t$, as follows:

$$
G_{d}(z)=\frac{Q_{d n s}(z)}{Q_{u p s}(z)}=\frac{b_{0} z^{-d}}{z-a_{1}},
$$

where $d=\tau_{d} / T_{s}, b_{0}=1-a_{1}$ and $a_{1}=e^{-\frac{T_{s}}{T}}$. Alternatively, it can be written as a difference equation as

$$
q_{d n s}(k+1)=a_{1} q_{d n s}(k)+b_{0} u(k-d) .
$$

5) Demand and irrigation sectors: Demand and irrigation sector represents the water demand made by the network users of a certain physical area. It is considered as a measured disturbance of the system at a given time instant. The demand in urban areas can be anticipated by a forecasting algorithm that is integrated within the MPC closed-loop architecture. The demand forecasting algorithm typically uses a two-level scheme composed by (i) a time-series model to represent the daily aggregate flow values, and (ii) a set of different daily flow demand patterns according to the day type to cater for different consumption during the weekends and holidays periods. Every pattern consists of 24 hourly values for each daily pattern [8]. This algorithm runs in parallel with the MPC algorithm. The daily series of hourly-flow predictions are computed as a product of the daily aggregate flow value and the appropriate hourly demand pattern. On the other hand, irrigation demand is typically planned in advance with farmers. Pre-established flows for irrigation are established in the irrigation areas in determined periods of the year.

\section{MPC PROBLEM Formulation FOR COMBINED IRRIGATION AND WATER SUPPLY SYSTEMS}

Water supply and distribution systems are very complex multivariate systems. In order to improve their performance, predictive optimal control [5] provides suitable techniques to compute optimal control strategies ahead in time for all the flow and pressure control elements of a water system. The optimal strategies are computed by optimizing a mathematical function describing the operational goals in a given time horizon and using a representative model of the network dynamics, as well as demand forecasts.

\section{A. Operational goals}

In most water systems, the regulated elements, namely pumps, gates and retention devices, are typically controlled locally, i.e., they are controlled by a remote station according to the measurements of sensors connected only to that station. However, a global RTC system requires the use of an operational model of the system dynamics in order to compute, ahead of time, optimal control strategies for the actuators based on the current state of the system provided by supervisory control and data acquisition (SCADA) sensors, the current disturbance measurements and appropriate disturbance predictions. The computation procedure of an optimal global control law should take into account all the physical and operational constraints of the dynamical system, producing set-points though which given control objectives are achieved.
The immediate control goal of a water supply system is to meet the demands at consumer sites with appropriate flows, according to users' needs. Predictive control techniques may be used to compute strategies which achieve this, while also optimizing the system performance in terms of different operational criteria, such as

1) Cost reduction: Water cost is usually related to acquisition, which may have different prices at different sources, and elevation, affected by power tariffs which may vary during the day.

2) Operational safety: This criterion refers to maintaining appropriate water storage levels in dams and reservoirs of the network for emergency-handling.

3) Quality management: This is especially important when several sources exist, with different quality levels and/or when quality refers to the concentration of a certain ion, such as chlorine, which decays in time.

4) Control actions smoothness for equipment conservation: The operation of water treatment plants and main valves usually requires smooth flow set-point variations.

5) Conservation and other policies: Water sources such as boreholes, reservoirs and rivers are usually subject to operational constraints to maintain water table levels, ecologic flows and water use sustainability.

\section{B. Non-linear Predictive Control Strategy}

Given the dynamic model of the water system

$$
x(k+1)=g(x(k), u(k)),
$$

being $x_{k} \in \mathbb{X} \subseteq \mathbb{R}^{n}$ the mapping of states and $u_{k} \in \mathbb{U} \subseteq \mathbb{R}^{m}$ the control signals, where $g: \mathbb{R}^{n} \times \mathbb{R}^{m} \rightarrow \mathbb{R}^{n}$ is an arbitrary system state function and $k \in \mathbb{Z}_{+}$, the MPC is based on the solution of the open-loop optimisation problem (OOP)

$$
\min _{\{u(k)\}_{i=0}^{H_{p}-1}} \sum_{i=0}^{H_{p}-1} J(u(k+i \mid k), x(k+i \mid k)),
$$

subject to

$$
\begin{aligned}
& H_{i q}^{\mathrm{u}} u(k) \leq b_{i q}^{\mathrm{u}}, \\
& G_{i q} x(k)+H_{i q} u(k) \leq b_{i q}, \\
& H_{e q}^{\mathrm{u}} u(k)=b_{e q}^{\mathrm{u}}, \\
& G_{e q} x(k)+H_{e q} u(k)=b_{e q},
\end{aligned}
$$

$\forall i \in\left[0, H_{p}-1\right]$, where $J(\cdot)$ is the cost function, $H_{p}$ denotes the prediction horizon or output horizon, and $G_{i q}, G_{\text {eqe }}$, $H_{i q}, H_{e q}, H_{i q}^{\mathrm{u}}, H_{e q}^{\mathrm{u}}, b_{i q}, b_{e q}, b_{i q}^{\mathrm{u}}$, and $b_{e q}^{\mathrm{u}}$ are matrices with suitable dimensions. In sequence (11a), $x(k+i \mid k)$ denotes the prediction of the state at time $k+i$ performed at $k$, starting from $x(0 \mid k)=x(k)$. When $H_{p}=\infty$, the OOP is called the infinite horizon problem; when $H_{p} \neq \infty$, the OOP is called the finite horizon problem. Constraints employed to guarantee the stability of the system in a closed loop would be added in (11b)-(11e). In particular, constraints (11d)-(11e) are related to elements with static dynamics, where an equality condition 
must hold. The optimal solution of the OOP (11) is given by the sequence

$$
u(0 \mid k)^{*}, u(1 \mid k)^{*}, \ldots, u\left(H_{p}-1 \mid k\right)^{*},
$$

and then the receding horizon philosophy sets

$$
u_{\mathrm{MPC}}(x(k)) \triangleq u(0 \mid k)^{*},
$$

and disregards the computed inputs from $k=1$ to $k=H_{p}-1$, with the whole process repeated at the next time instant $k \in$ $\mathbb{Z}_{+}$. Expression (12) is known in the MPC literature as the MPC law.

\section{Case Study: Guadiana River}

\section{A. Description}

The Guadiana is the fourth longest river in Spain and the tenth larger. It has its source in Spain and flows into the Atlantic Ocean on the Portugal coast. It runs through the central south part of Spain from east to west most of its length to turn to the south direction when it is passing through Portugal.The Hydrologic basin of Guadiana covers up to $67733 \mathrm{~km}^{2}$, from which $55512 \mathrm{~m}^{2}$ belongs to Spain $(81.9 \%)$. The annual average sources to the basin is about $3884 \mathrm{hm}^{3}$. The global basin has 87 reservoirs with higher capacity than $1 \mathrm{hm}^{3}$ and the total storage capacity is more than $9000 \mathrm{~m}^{3}$.

This paper is focused on the central part of this basin, where the Guadiana River is highly regulated by 16 dams linked by channels and pipes. The total capacity of these reservoirs is about $7800 \mathrm{hm}^{3}$, which regulate the river flow and supply to all the demands and the important irrigation zones of the basin. The transaction between the two countries (Spain and Portugal) is regulated through the Convenio de Albufeira, which defines the volumes and flows to supply during the year. This regulation takes into account the restrictions of ecological flows for summer periods and the operational rules for the reservoirs in rainy episodes. All these restrictions will be part of the optimization problem. The conceptual model of the Guadiana central part zone is shown in Figure 1 that allows to see that it is a large-scale complex system. In this figure, blue lines define the stretches rivers and the open channels, while in black lines are defined the pressurized pipes.

\section{B. Control objectives}

The control objectives of the multi-objective optimization associated to the MPC problem are defined as follows:

- Satisfy all the demands and irrigations.

- Respect the environmental flows in rivers and minimum volumes at reservoirs.

- Maintain the water levels inside the operational intervals, avoiding river flooding.

- Generate smooth control signal variations.
The total cost function in the MPC problem (11) that brings all these goals together is as follows:

$$
\begin{array}{r}
J(k)=\omega_{1} \sum_{i=1}^{p} V_{\sec _{i}}(k)+\omega_{2} \sum_{i=1}^{q} Q_{\sec _{i}}(k)+ \\
\omega_{3} \sum_{i=1}^{r} Q_{\text {full }_{i}}(k)+\omega_{4} \sum_{i=1}^{s} Q_{\operatorname{stab}_{i}}(k),
\end{array}
$$

where the first term is related to the safety volume in the $p$ reservoirs; second term is related to penalties in the flooding and in the environmental flow for the $q$ river reaches; third term is related to the penalty term of not fulfilling the flow required in the $r$ irrigation areas; and the fourth term is related to the smoothness of the $s$ control actions. Constants $\omega_{i}$ are the weighting factors used in the prioritisation of the different control objectives.

\section{Results}

In this section, control results obtained in a year and four month long scenario are presented for representative selected elements. The control results have been obtained under the following considerations:

- The considered scenario lasts a year and four months and the sampling time is one day.

- The operational control strategy aim is to guarantee the preservation of a minimum volume of water at the reservoirs and the smooth behaviour of the actuators (gates) while satisfying the consumer and irrigation demands and the Convenio of Albufeira.

- The maximum available flow to deliver through all outlets of the reservoirs is limited by the total volume of water according to the corresponding discharge curve.

The prediction horizon uses for the MPC controller is one month (30 days) while the sampling time is one day. Simulations have been carried out using the CONOPT3 solver in GAMS. The computer used to run the simulations is a PC Intel $^{\circledR}$ Core $^{\mathrm{TM}}$ running both cores at $2.8 \mathrm{GHz}$ with $4 \mathrm{~GB}$ of RAM.

Figure 2 shows the water demand E2-07 for supply and irrigation in a typical scenario. Figure 3 shows the resulting flow through the gate V2-07 to satisfy such a demand. At the same time, the control strategy guarantees that the dam volumes are over their safety volume most of the time, being able to vary freely but without getting empty. This can be seen in Figure 4 for the volume evolution of dam E2-07. Notice also that the maximum available flow delivered at the output of dam through all is limited by the total volume of water according to the discharge curve.

\section{Conclusions}

In this paper, a methodology for the optimal management of a combined irrigation and water supply system based on model predictive control (MPC) has been proposed. A controloriented modelling methodology for this type of systems has also been presented. MPC has been used to generate flow control strategies from the sources to the consumer 


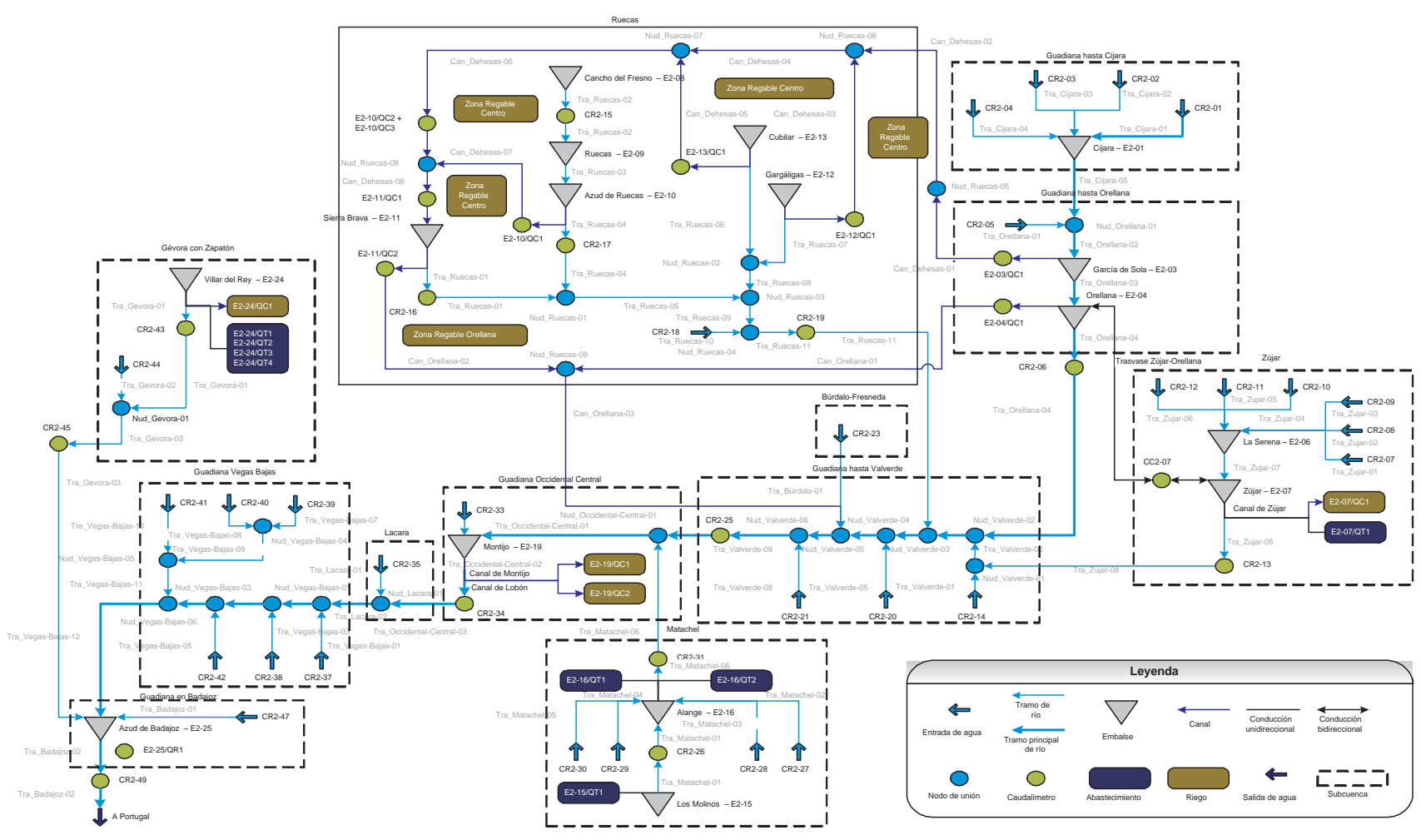

Figure 1. Conceptual scheme of the Guadiana central part used as case study

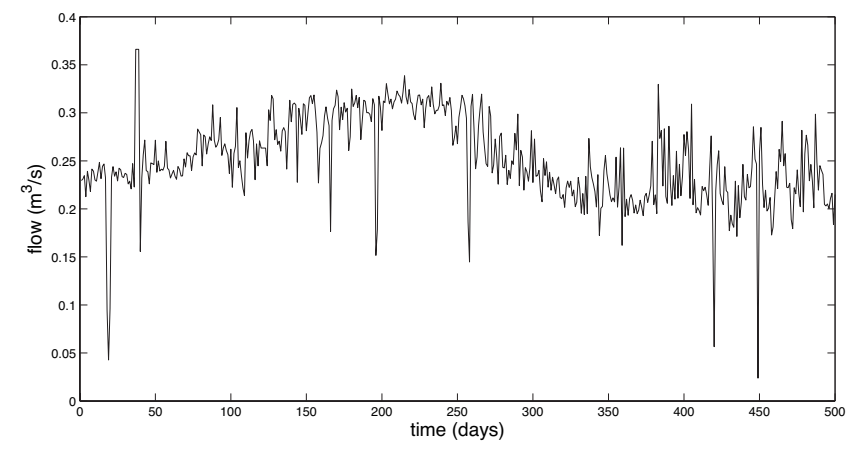

Figure 2. Water demand E2-07

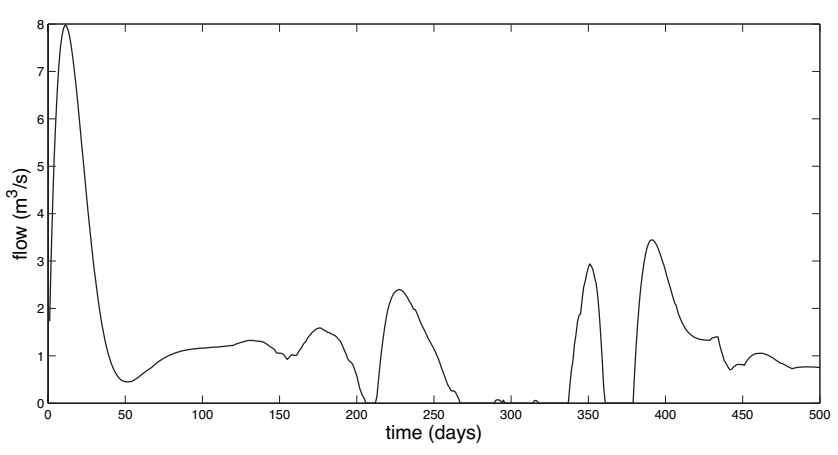

Figure 3. Flow through gate V2-07

and irrigation areas to meet future demands with appropriate flows, optimizing operational goals such as network safety

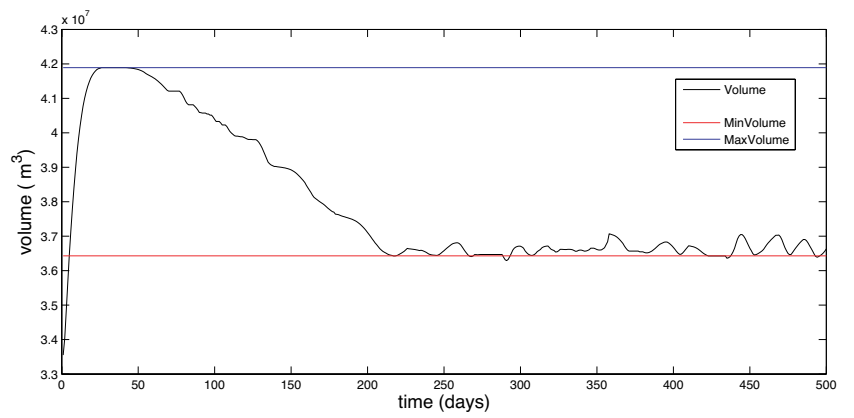

Figure 4. Volume evolution of dam E2-07

volumes in dams, ecological flows in rivers and flow control stability in actuators. The case study of Guadiana River has been used to exemplify and verify the proposed management methodology. Results have shown the effectiveness of the proposed modelling and control methodologies.

\section{ACKNOWLEDGMENTS}

This research has been partially founded by CDTI (MCyT) project HIDROPTIM IDI-20100722, the Spanish research project WATMAN (DPI2009-13744) of the Science and Technology Ministry and the DGR of Generalitat de Catalunya (SAC group Ref. 2009/SGR/1491). The authors thank Confederación Hidrográfica del Guadiana for providing the case study as well as for sharing their hydrological management expertise. 


\section{REFERENCES}

[1] M. Brdys and B. Ulanicki. Operational Control of Water Systems: Structures, algorithms and applications. Prentice Hall International, UK, 1994.

[2] G. Cembrano, J. Quevedo, V. Puig, R. Pérez, J. Figueras, J. M. Verdejo, I. Escaler, G. Ramón, G. Barnet, P. Rodríguez, and M. Casas. Plio: A generic tool for real-time operational predictive optimal control of water networks. Water Science and Technology (to appear), 2011.

[3] G. Cembrano, J. Quevedo, M. Salamero, V. Puig, J. Figueras, and J. Martí. Optimal control of urban drainage systems: a case study. Control Engineering Practice, 12(1):1-9, 2004.

[4] X. Litrico and V. Fromion. Simplified modeling of irrigation canals for controller design. Journal of Irrigation and Drainage Engineering, 130(5):373 - 383, 2004.

[5] J.M. Maciejowski. Predictive Control with Constraints. Prentice Hall, Great Britain, 2002.

[6] L.W. Mays. Urban Stormwater Management Tools. McGrawHill Professional Publishing, USA, 2004.

[7] C. Ocampo-Martinez, V. Puig, G. Cembrano, R. Creus, and M. Minoves. Improving water management efficiency by using optimization-based control strategies: the Barcelona case study. Water Science \& Technology: Water supply, 9(5):565-575, 2009.

[8] J. Quevedo, V. Puig, G. Cembrano, and J. Blanch. Validation and reconstruction of flow meter data in the Barcelona water distribution network. Control Engineering Practice, 11(6):640-651, June 2010.

\section{APPENDIX: HYDROPTIM TOOL}

\section{A. Overview}

One the main results of the project described in this paper is the development of the HYDROPTIM decision support tool. HYDROPTIM is a graphical real-time decision support tool for integral operational planning of water systems. HYDROPTIM will be developed using standard GUI (graphical user interface) techniques and object oriented programming. HYDROPTIM calls a commercial solver to determine the optimal solutions of the optimization problem associated to the predictive optimal control using nonlinear programming techniques.

\section{B. HYDROPTIM operating modes}

The tool has four modes of operation: edition, simulation, monitoring and reproduction modes:

1) Edition mode: This mode allows graphically building and parameterizing the network using a palette of building blocks, defining the control objectives and generating the optimization model equations. HYDROPTIM has different element libraries which allow the user to easily model the network. Elements include reservoirs, tanks, water demands, sensors and actuators. The user may place these elements in the model using drag and drop and then connect using pipes, aqueducts, etc. Each element in HYDROPTIM has a number of properties, which are grouped in trees. These identify the element, parameterize its characteristics, provide goals to the optimizer, define SCADA data links and database presence, etc. Once the network has been built, HYDROPTIM tests it for consistency and creates the set of optimization equations using the goals and constraints defined in each element.

2) Simulation (or off-line) mode: This mode allows network optimization in simulation, using the demands from the HYDROPTIM database corresponding to a recorded real scenario as inputs, HYDROPTIM generates the optimal controls, which are applied to the same network model (as a substitute of the real network). Graphical evolution of the main network variables and controls can be represented and registered in HYDROPTIM database for further study.

3) Monitoring (or on-line) mode: Network optimization in real time is carried out in monitoring mode, using the demands and measurements from network real state coming from the telemetry system, provided by the SCADA system. HYDROPTIM generates the optimal controls, which are applied to the real network only after confirmation by an operator. Graphical evolution of the main network variables and controls can be represented and registered in HYDROPTIM database for further study.

4) Reproduction mode: This mode allows the reproduction of network state evolution under specified operation conditions and control set-points (optimal or other). HYDROPTIM will provide a graphical representation of the main variable evolution in a real or simulated scenario. 